New Mexico Tech (October 26, 2018)

\title{
Noncommutative Einstein Equations
}

\author{
Guglielmo Fucci and Ivan G. Avramidi \\ New Mexico Institute of Mining and Technology \\ Socorro, NM 87801, USA \\ E-mail: iavramid@nmt.edu,gfucci@nmt.edu
}

We study a noncommutative deformation of general relativity where the gravitational field is described by a matrix-valued symmetric twotensor field. The equations of motion are derived in the framework of this new theory by varying a diffeomorphisms and gauge invariant action constructed by using a matrix-valued scalar curvature. Interestingly the genuine noncommutative part of the dynamical equations is described only in terms of a particular tensor density that vanishes identically in the commutative limit. A noncommutative generalization of the energy-momentum tensor for the matter field is studied as well. 


\section{Introduction}

The basic notions of General Relativity (GR) are closely related with the geometric interpretation of second order elliptic partial differential operators describing the propagation of massless scalar fields without internal structure [1]. Let us consider a manifold $M$ without boundary. Let $f \in C^{\infty}(M)$ be a smooth function on $M$ and let $L: C^{\infty}(M) \longrightarrow C^{\infty}(M)$ be a second order partial differential operator of the form

$$
L=-a^{\mu \nu}(x) \partial_{\mu} \partial_{v}+b^{\mu}(x) \partial_{\mu}+c(x),
$$

with smooth real coefficients $a^{\mu \nu}, b^{\mu}$ and $c$. In particular, $a^{\mu \nu}$ is a real smooth symmetric non-degenerate matrix at each point of the manifold $M$, that is, for any point $x$ in $M$

$$
a^{\mu \nu}(x)=a^{\nu \mu}(x), \quad \operatorname{det} a^{\mu \nu}(x) \neq 0 .
$$

Since the matrix $a^{\mu \nu}$ is symmetric, it has real eigenvalues and since the determinant is different from zero through the whole manifold $M$, it has constant signature. If the number of negative eigenvalues is 1 , the operator $L$ is called hyperbolic. Hyperbolic operators defined on $M$ describe the propagation of massless scalar fields, in particular photons, over the manifold $M$.

It can be easily shown that for the operator $L$ to be covariant the matrix $a^{\mu \nu}$ should transform like a tensor of type $(2,0)$ under diffeomorphisms of the manifold $M$. This enables us to identify the matrix $a^{\mu \nu}$ with a Riemannian metric $g^{\mu \nu}$ over $M$. Once a metric $g^{\mu \nu}$ is defined one can construct all the geometrical quantities needed to develop the theory of General Relativity. In this theory the gravitational field is described by a symmetric non-degenerate tensor of type $(0,2)$ and its dynamics is described by Einstein's equations.

A natural generalization of the GR theory leads us to consider the gravitational field described by a $(N \times N)$ matrix of metrics $\left(g^{\mu \nu}\right)_{B}^{A}$ instead of a single metric (for details see [1, 2]). Such a matrix-valued metric appear naturally if one considers the propagation of a field with an internal structure (a multiplet of fields rather than a single field). In this case the coefficients of the operator $\mathrm{L}$, that is, the functions $a^{\mu \nu}(x), b^{\mu}(x)$ and $c(x)$, become matrix-valued. This follows from the idea that at short distances, or at high energies, the role played by the photon can be played by a multiplet of gauge fields [1].

It is important to stress here that this approach for deforming general relativity is different from the ones proposed in the framework of noncommutative geometry [5, 6, 7, 9], where the coordinates do not commute and the standard product between functions is replaced by the Moyal product [8]. 
The purpose of this paper is to derive the equations of motion for the field $a^{\mu \nu}$ that generalizes the role played by $g^{\mu v}$ in the general theory of relativity. Since this model is a noncommutative extension of Einstein's general relativity we will call the corresponding equations of motions noncommutative Einstein's equations.

The outline of the paper is as follows. First we develop the necessary formalism that will be used in the derivation of the dynamical equations of the theory. More precisely we will describe a generalization of various important geometrical object to endomorphism-valued quantities. Then we construct an action that is invariant under diffeomorphisms of the manifold $M$ and gauge transformations and contains the matrix-valued scalar curvature. By varying, then, the action with respect to the new dynamical field $a^{\mu \nu}$, we obtain the equations of motion.

\section{Matrix Geometry}

We will describe, in this section, the formalism needed in order to write the action for matrix general relativity following [1, 2] and derive the equations of motion of the theory. This formalism is a generalization of differential geometry, which is the natural language of general relativity, and it will be called Matrix Geometry. The formalism that we are going to describe is related to the algebra-valued formulation of Mann [3] and Wald [4].

As already stated before, the main idea is to describe the gravitational field as a matrix-valued symmetric two-tensor field. Let $M$ be an $n$-dimensional Riemannian manifold without boundary and let $g^{\mu \nu}$ be a metric tensor defined on the tangent bundle $T M$. It is well known that General Relativity is nothing but the dynamical theory of the metric 2-tensor field which is, basically, an isomorphism between tangent and cotangent bundles. In our model the metric 2-tensor field is replaced by a endomorphism-valued 2-tensor field $a^{\mu \nu}$ which represents an isomorphism of more general bundles over the manifold $M$. The main idea here, similar to general relativity, is to develop a dynamical theory of this endomorphismvalued 2-tensor field $a^{\mu \nu}$. This generality brings a much richer structure and content to the model.

Let $V$ be an $N$-dimensional Hermitian vector bundle over $M$, let $\mathscr{T}=T M \otimes V$ be the bundle constructed by taking the tensor product of the tangent bundle to the manifold $M$ with the vector bundle $V$, and let $\mathscr{T}^{*}=T^{*} M \otimes V$, where $T^{*} M$ is the cotangent bundle to $M$. We consider, then, the bundle $\operatorname{Iso}\left(\mathscr{T}, \mathscr{T}^{*}\right)$, elements of which, are isomorphisms between $\mathscr{T}$ and $\mathscr{T}^{*}$. In general the isomorphisms $B: \mathscr{T} \longrightarrow \mathscr{T}^{*}$ can be identified with the sections of the bundle $T^{*} M \otimes T^{*} M \otimes$ 
End $(V)$ and the isomorphisms $A: \mathscr{T}^{*} \longrightarrow \mathscr{T}$ with the sections of the bundle $T M \otimes T M \otimes \operatorname{End}(V)$.

Let $a$ be a symmetric self-adjoint element of $T M \otimes T M \otimes \operatorname{End}(V)$, that is,

$$
a^{\mu \nu}=a^{\nu \mu}, \quad\left(a^{\mu \nu}\right)^{*}=a^{\mu \nu} .
$$

This element is an isomorphism between the bundles $\mathscr{T}^{*}$ and $\mathscr{T}$ if the following equation

$$
a^{\mu v} \phi_{v}=\psi^{\mu},
$$

with arbitrary $\psi \in \mathscr{T}^{*}$, has a unique solution

$$
\phi_{v}=b_{v \mu} \psi^{\mu} .
$$

This last requirement can be cast in another form; the element $a$ is an isomorphism if the following equation

$$
a^{\mu \nu} b_{\nu \rho}=b_{\mu \nu} a^{\nu \rho}=\delta_{\mu}^{\rho} \cdot \mathbb{I},
$$

has a unique solution $b \in T^{*} M \otimes T^{*} M \otimes \operatorname{End}(V)$.

There are some properties of the matrix $b_{\mu \nu}$ that need attention. The first property is the following: the matrix $b_{\mu \nu}$ satisfies the following equation

$$
b_{\mu \nu}^{*}=b_{\nu \mu},
$$

but it is not necessarily a self-adjoint matrix symmetric in its tensor indices. Moreover, one can use $a^{\mu \nu}$ and $b_{\mu \nu}$ to lower and raise indices, although particular care is required in this operations because, in general, $a^{\mu \nu}$ and $b_{\mu \nu}$ do not commute and $b_{\mu \nu}$ is not symmetric in its tensorial indices [1].

We need, now, to develop some kind of invariant calculus. For this purpose, we introduce matrix-valued Christoffel symbols $\mathscr{A}^{\mu}{ }_{\alpha \beta}$ that transform, under diffeomorphisms of $M$, as connection coefficients, namely

$$
\mathscr{A}^{\prime \mu^{\prime}}{ }_{\alpha^{\prime} \beta^{\prime}}\left(x^{\prime}\right)=\frac{\partial x^{\prime \mu}}{\partial x^{v}} \frac{\partial x^{\gamma}}{\partial x^{\prime \alpha}} \frac{\partial x^{\delta}}{\partial x^{\prime \beta}} \mathscr{A}^{\nu}{ }_{\gamma \delta}(x)+\frac{\partial x^{\prime \mu}}{\partial x^{\nu}} \frac{\partial^{2} x^{\nu}}{\partial x^{\prime \alpha} \partial x^{\prime \beta}} \cdot \mathbb{I},
$$

where $\mathbb{I}$ is the identity matrix. Once we have a connection on $M$ we can define a way to differentiate tensors.

Let $T_{p}^{q}(M)$ be the tensor bundle of type $(p, q)$ on the manifold $M$. We define the following linear map

$$
\mathcal{D}: T_{p}^{q} \otimes V \longrightarrow T_{p}^{q+1} \otimes V
$$


by

$$
\mathcal{D}_{\alpha} \phi_{v_{1} \ldots v_{q}}^{\mu_{1} \ldots \mu_{p}}=\partial_{\alpha} \phi_{v_{1} \ldots v_{q}}^{\mu_{1} \ldots \mu_{p}}+\sum_{j=1}^{p} \mathscr{A}_{\lambda \alpha}^{\mu_{j}} \phi_{v_{1} \ldots v_{q}}^{\mu_{1} \ldots \mu_{j-1} \lambda \mu_{j+1} \ldots \mu_{p}}-\sum_{i=1}^{q} \mathscr{A}_{v_{i} \alpha}^{\lambda} \phi_{v_{1} \ldots v_{i-1} \lambda v_{i+1} \ldots v_{q}}^{\mu_{1} \ldots \mu_{p}} .
$$

This map is a well defined operator between $T_{p}^{q} \otimes V$ and $T_{p}^{q+1} \otimes V$. It is important to stress, at this point, that the linear map defined in (2.9) is not a covariant differentiation. In fact, it is easy to show that, because of the non-commutativity, the Leibnitz rule does not hold. However, in the commutative limit, the operator (2.8) reduces to an ordinary covariant derivative on the manifold $M$.

In complete analogy with the ordinary Riemannian geometry, we shall define the matrix curvature and the matrix torsion tensors. Let $\phi \in T^{*} M \otimes V$, we compute

$$
\mathcal{D}_{\mu} \mathcal{D}_{\nu} \phi_{\alpha}-\mathcal{D}_{\nu} \mathcal{D}_{\mu} \phi_{\alpha}=-\mathcal{R}^{\lambda}{ }_{\alpha \mu \nu} \phi_{\lambda}+\mathcal{T}^{\lambda}{ }_{\mu \nu} \mathcal{D}_{\lambda} \phi_{\alpha}
$$

where

$$
\mathcal{R}_{\alpha \mu \nu}^{\lambda}=\partial_{\mu} \mathscr{A}^{\lambda}{ }_{\alpha \nu}-\partial_{v} \mathscr{A}^{\lambda}{ }_{\alpha \mu}+\mathscr{A}_{\beta \mu}^{\lambda} \mathscr{A}^{\beta}{ }_{\alpha \nu}-\mathscr{A}_{\beta v}^{\lambda} \mathscr{A}^{\beta}{ }_{\alpha \mu},
$$

and

$$
\mathcal{T}^{\lambda}{ }_{\mu \nu}=\mathscr{A}^{\lambda}{ }_{\mu \nu}-\mathscr{A}^{\lambda}{ }_{\nu \mu} .
$$

Once the matrix curvature (Riemann) tensor is defined we can construct the matrix Ricci tensor, namely

$$
\mathcal{R}_{\mu \nu}=\mathcal{R}_{\mu \alpha v}^{\alpha} .
$$

In order to write the action for matrix gravity, we need to introduce the matrix scalar curvature $\mathcal{R}$. Since the metric $a^{\mu \nu}$ and the Ricci tensor $\mathcal{R}_{\mu \nu}$ are matrices, they do not commute in general and the definition of the scalar curvature, obtained by contracting the metric tensor with the Ricci tensor from the left, would be different if the contraction would be performed with the metric tensor on the right. In order to avoid this choice, we use a symmetrized definition of the matrix-valued scalar curvature as follows

$$
\mathcal{R}=\frac{1}{2}\left(a^{\mu v} \mathcal{R}_{\mu \nu}+\mathcal{R}_{\mu \nu} a^{\mu \nu}\right) .
$$

We need, now, to relate the connection coefficients $\mathscr{A}^{\rho}{ }_{\mu \nu}$ to the metric tensor $a^{\mu \nu}$. We impose a compatibility condition similar to the one in Riemannian geometry as

$$
\partial_{\mu} a^{\alpha \beta}+\mathscr{A}_{\lambda \mu}^{\alpha} a^{\lambda \beta}+\mathscr{A}_{\lambda \mu}^{\beta} a^{\alpha \lambda}=0 .
$$

The above equation has the following solution in a closed form [1]

$$
\mathscr{A}_{\lambda \mu}^{\alpha}=\frac{1}{2} b_{\lambda \sigma}\left(a^{\alpha \gamma} \partial_{\gamma} a^{\rho \sigma}-a^{\rho \gamma} \partial_{\gamma} a^{\sigma \alpha}-a^{\sigma \gamma} \partial_{\gamma} a^{\alpha \rho}+S^{\alpha \rho \sigma}+S^{\rho \sigma \alpha}+S^{\sigma \rho \alpha}\right) b_{\rho \mu},
$$


where $S$ is an arbitrary matrix-valued tensor that satisfies the symmetry property

$$
S^{\alpha \mu \nu}=-S^{\alpha \nu \mu} .
$$

The matrix-valued tensor $S$ is related, in the general case, with the torsion $\mathcal{T}$ in (2.12). Moreover, in the commutative limit, the tensor $S$ reduces exactly to the torsion. It is important to notice that in matrix geometry the connection (2.16) is not symmetric in the two lower indices even if the tensor $S$ vanishes. In the rest of the paper we will assume, without loss of generality, that the tensor $S$ vanishes.

In order to write an action for the model under consideration we need a generalization of the concept of measure. As a guiding principle, any generalization of the measure $\mu$ has to lead, in the commutative limit, to the ordinary Riemannian measure $\sqrt{\left|\operatorname{det} g_{\mu \nu}\right|}$. Moreover the measure $\mu$ is a density depending only on the metric $a^{\mu \nu}$ and not on its derivatives and transforming, under diffeomorphisms of $M$, as

$$
\mu^{\prime}\left(x^{\prime}\right)=J(x) \mu(x)
$$

where

$$
J(x)=\operatorname{det}\left(\frac{\partial x^{\prime \mu}(x)}{\partial x^{\alpha}}\right) .
$$

A definition of the measure $\mu$ which is a straightforward generalization of the Riemannian measure, is the following [1]

$$
\mu=\frac{1}{N} \operatorname{Tr}_{V} \rho,
$$

where $\rho$ is a matrix-valued scalar density, which can be defined, for example, as follows

$$
\rho=\int_{\mathbb{R}^{n}} \frac{d \xi}{\pi^{\frac{n}{2}}} \exp \left(-a^{\mu v} \xi_{\mu} \xi_{v}\right) .
$$

Then $\rho$ only depends on the metric $a$ and transforms in the correct way under diffeomorphisms of $M$.

\section{Variation of the Action}

We construct the action functional for the field $a^{\mu \nu}$ following [1]. This functional has to be invariant under both diffeomorphisms of $M$ and gauge transformations. The infinitesimal form of these transformations is

$$
\delta_{\omega} a^{\mu \nu}=\left[\omega, a^{\mu \nu}\right]
$$


and

$$
\delta_{\xi} a^{\mu \nu}=\mathscr{L}_{\xi} a^{\mu \nu}
$$

where $\omega$ is an element of the algebra of the gauge group

$$
\delta_{\omega} U=\omega
$$

and $\xi$ is the generator of the infinitesimal coordinate transformation

$$
\delta_{\xi} x^{\mu}=\mathscr{L}_{\xi} x^{\mu}=-\xi^{\mu} .
$$

We can construct an action functional for field $a^{\mu \nu}$ that satisfies the properties described above, by using the matrix-valued scalar curvature, defined in (2.14), and the measure (2.20). A good candidate for the action is the following

$$
S_{\mathrm{MGR}}(a)=\frac{1}{16 \pi G} \int_{M} d x \frac{1}{N} \operatorname{Tr}_{V}(\rho \mathcal{R}) .
$$

It is worth noticing that because of the cyclic property of the trace, the relative position of $\rho$ and the scalar curvature is irrelevant, moreover it is easily shown that the action functional (3.24) is invariant under the diffeomorphisms (3.23) and under the gauge transformations (3.22). Since the action has the invariant properties discussed above, the currents associated with the symmetries satisfy the identities

$$
\partial_{\alpha}\left(a^{\mu \alpha} \frac{\delta S}{\delta a^{\mu \lambda}}\right)+\frac{1}{2}\left(\partial_{\lambda} a^{\mu \nu}\right) \frac{\delta S}{\delta a^{\mu \nu}}=0
$$

and

$$
\left[a^{\mu v}, \frac{\delta S}{\delta a^{\mu \nu}}\right]=0
$$

where the (3.25) is the current generated by the invariance with respect to diffeomorphisms and (3.26) is the current generated by the internal (gauge) symmetry. The above identities represent an endomorphism-valued generalization of the Noëther identities, which are related, in the usual theory, to the contracted Bianchi identities.

Now that we have the action functional we can derive the equations of motion for the field $a^{\mu v}$, which is the main goal and the main result of the present paper. These equations will be matrix-valued and they will constitute a generalization of the ordinary Einstein's equations that we will call noncommutative Einstein equations. In order to find the dynamics of the model we vary the action (3.24) with respect to the field $a^{\mu \nu}$ considered as independent variable, namely

$$
a^{\mu \nu} \longrightarrow a^{\mu \nu}+\delta a^{\mu \nu}
$$


By doing so we obtain, for the variation of the action, the following

$$
\delta S=S\left(a^{\mu \nu}+\delta a^{\mu \nu}\right)-S\left(a^{\mu \nu}\right)=\frac{1}{16 \pi G} \int_{M} d x \frac{1}{N} \operatorname{Tr}_{V}\left(\mathcal{G}_{\mu \nu} \delta a^{\mu \nu}\right),
$$

where $\mathcal{G}_{\mu \nu}$ is some matrix valued symmetric tensor density. Then, of course, the desired equations of motion are

$$
\mathcal{G}_{\mu \nu}=0 .
$$

It is important to notice that the matrix-valued tensor density (3.28) has to coincide with the Einstein tensor in the commutative limit, more precisely we need that, in the commutative limit, the following relation holds

$$
\sqrt{g}\left(R_{\mu \nu}-\frac{1}{2} g_{\mu \nu} R\right)=\frac{1}{N} \operatorname{Tr} \mathcal{G}_{\mu \nu} .
$$

Our main task, then, is to find the explicit form of the equations of motion that result from the variation of the action (3.24). In all the calculations that will follow the order of the terms is important, unless explicitly stated, due to the matrix nature of them.

First of all, we rewrite the action in a more explicit form which is more suitable for the subsequent variation, namely

$$
S_{\mathrm{MGR}}(a)=\frac{1}{16 \pi G} \int_{M} d x \frac{1}{N} \operatorname{Tr}_{V}\left[\rho \frac{1}{2}\left(a^{\mu v} \mathcal{R}_{\mu \nu}+\mathcal{R}_{\mu \nu} a^{\mu \nu}\right)\right] .
$$

By varying the terms in (3.30) with respect to the independent field $a^{\mu \nu}$, and by using the cyclic property of the trace we get

$$
\delta S_{\mathrm{MGR}}(a)=\frac{1}{16 \pi G} \int_{M} d x \frac{1}{N} \operatorname{Tr}_{V}\left[\delta \rho \mathcal{R}+\frac{1}{2}\left\{\mathcal{R}_{\mu \nu}, \rho\right\} \delta a^{\mu \nu}+\frac{1}{2}\left\{\rho, a^{\mu \nu}\right\} \delta \mathcal{R}_{\mu \nu}\right],
$$

where the curly brackets $\{$,$\} denote anti-commutation, namely \{A, B\}=A B+B A$.

From the expressions (2.11) and (2.13), we can evaluate the variation of the matrix-valued Ricci tensor, more precisely we have

$$
\begin{aligned}
\delta \mathcal{R}_{\mu \nu} & =\partial_{\alpha}\left(\delta \mathscr{A}^{\alpha}{ }_{\mu \nu}\right)-\partial_{v}\left(\delta \mathscr{A}^{\alpha}{ }_{\mu \alpha}\right)+\delta \mathscr{A}^{\alpha}{ }_{\lambda \alpha} \mathscr{A}^{\lambda}{ }_{\mu \nu}+\mathscr{A}^{\alpha}{ }_{\lambda \alpha} \delta \mathscr{A}^{\lambda}{ }_{\mu \nu}+ \\
& -\delta \mathscr{A}^{\alpha}{ }_{\lambda \nu} \mathscr{A}^{\lambda}{ }_{\mu \alpha}-\mathscr{A}^{\alpha}{ }_{\lambda \nu} \delta \mathscr{A}^{\lambda}{ }_{\mu \alpha} .
\end{aligned}
$$

From now on, for simplicity of notation, we set

$$
B^{\mu v} \equiv\left\{\rho, a^{\mu \nu}\right\} .
$$


By substituting (3.32) in (3.31), and by using the cyclic property of the trace we obtain

$$
\begin{aligned}
\delta S_{\mathrm{MGR}}(a) & =\frac{1}{16 \pi G} \int_{M} d x \frac{1}{N} \operatorname{Tr}_{V}\left[\delta \rho \mathcal{R}+\frac{1}{2}\left\{\mathcal{R}_{\mu \nu}, \rho\right\} \delta a^{\mu \nu}+\frac{1}{2} B^{\mu v} \partial_{\alpha}\left(\delta \mathscr{A}^{\alpha}{ }_{\mu \nu}\right)+\right. \\
& -\frac{1}{2} B^{\mu v} \partial_{\nu}\left(\delta \mathscr{A}^{\alpha}{ }_{\mu \alpha}\right)+\frac{1}{2} \mathscr{A}_{\mu \nu}^{\lambda} B^{\mu v} \delta \mathscr{A}^{\alpha}{ }_{\lambda \alpha}+\frac{1}{2} B^{\mu v} \mathscr{A}^{\alpha}{ }_{\lambda \alpha} \delta \mathscr{A}^{\lambda}{ }_{\mu \nu}+ \\
& \left.-\frac{1}{2} \mathscr{A}^{\lambda}{ }_{\mu \alpha} B^{\mu v} \delta \mathscr{A}^{\alpha}{ }_{\lambda \nu}-\frac{1}{2} B^{\mu v} \mathscr{A}^{\alpha}{ }_{\lambda \nu} \delta \mathscr{A}^{\lambda}{ }_{\mu \alpha}\right] .
\end{aligned}
$$

By integrating by parts and by collecting similar terms we get

$$
\begin{aligned}
\delta S_{\mathrm{MGR}}(a) & =\frac{1}{16 \pi G} \int_{M} d x \frac{1}{N} \operatorname{Tr}_{V}\left[\delta \rho \mathcal{R}+\frac{1}{2}\left\{\mathcal{R}_{\mu \nu}, \rho\right\} \delta a^{\mu \nu}-\frac{1}{2}\left(B^{\mu \nu}{ }_{, \alpha}-B^{\mu \nu} \mathscr{A}_{\alpha \lambda}^{\lambda}+\right.\right. \\
& \left.\left.+\mathscr{A}^{\mu}{ }_{\lambda \alpha} B^{\lambda \nu}+B^{\mu \lambda} \mathscr{A}^{\nu}{ }_{\alpha \lambda}\right) \delta \mathscr{A}^{\alpha}{ }_{\mu \nu}+\frac{1}{2}\left(B^{\mu v}{ }_{, \nu}+\mathscr{A}^{\mu}{ }_{\lambda \nu} B^{\lambda v}\right) \delta \mathscr{A}^{\alpha}{ }_{\mu \alpha}\right] .(3.35)
\end{aligned}
$$

We can rewrite the last expression in a more compact form, namely

$\delta S_{\mathrm{MGR}}(a)=\frac{1}{16 \pi G} \int_{M} d x \frac{1}{N} \operatorname{Tr}_{V}\left[\delta \rho \mathcal{R}+\frac{1}{2}\left\{\mathcal{R}_{\mu \nu}, \rho\right\} \delta a^{\mu \nu}-\frac{1}{2} C^{\mu \nu}{ }_{\alpha} \delta \mathscr{A}^{\alpha}{ }_{\mu \nu}+\frac{1}{2} D^{\mu} \delta \mathscr{A}^{\alpha}{ }_{\mu \alpha}\right]$,

where the matrix-valued tensor densities $C^{\mu v}{ }_{\alpha}$ and $D^{\mu}$ have the explicit expression

$$
\begin{aligned}
C^{\mu \nu}{ }_{\alpha} & =\left\{a^{\mu \nu}, \rho_{, \alpha}-\rho \mathscr{A}^{\lambda}{ }_{\alpha \lambda}\right\}-\rho\left[a^{\mu \nu}, \mathscr{A}^{\lambda}{ }_{\alpha \lambda}\right]-\left[\rho, \mathscr{A}^{\mu}{ }_{\rho \alpha}\right] a^{\rho \nu}+ \\
& -\left\{\rho,\left[\mathscr{A}^{v}{ }_{\lambda \alpha}, a^{\mu \lambda}\right]\right\}-a^{\mu \lambda}\left[\mathscr{A}^{v}{ }_{\lambda \alpha}, \rho\right]+2\left\{\rho, a^{\mu \lambda}\right\} \mathscr{A}^{v}{ }_{[\alpha \lambda]},
\end{aligned}
$$

and

$$
D^{\mu}=\left\{a^{\mu v}, \rho_{, v}-\mathscr{A}^{\rho}{ }_{\nu \rho} \rho\right\}-\left[\rho, \mathscr{A}^{\mu}{ }_{\rho v}\right] a^{\rho v}-\left[\rho, \mathscr{A}^{\rho}{ }_{\nu \rho}\right] a^{\mu \nu} .
$$

It is worth noticing that in the commutative limit, or, in other words, when all the matrices commute, the tensor densities $C^{\mu v}{ }_{\alpha}$ and $D^{\mu}$ are identically zero, and the variation of the action $\delta S_{\text {MGR }}$ simply reduces to the standard result of the general theory of relativity.

We can write, now, the variation of the connection coefficients. By using the expression (2.16), and by noticing that

$$
\delta b_{\mu \nu}=-b_{\mu \rho}\left(\delta a^{\rho \sigma}\right) b_{\sigma v}
$$


we obtain the following

$$
\begin{aligned}
\delta \mathscr{A}_{\lambda \mu}^{\alpha} & =-b_{\lambda \nu} \delta a^{\nu \beta} \mathscr{A}_{\beta \mu}^{\alpha}-\mathscr{A}_{\lambda \nu}^{\alpha} \delta a^{\nu \beta} b_{\beta \mu}+\frac{1}{2} b_{\lambda \sigma} \delta a^{\alpha \gamma}\left(\partial_{\gamma} a^{\rho \sigma}\right) b_{\rho \mu}+ \\
& -\frac{1}{2} b_{\lambda \sigma} \delta a^{\rho \gamma}\left(\partial_{\gamma} a^{\sigma \alpha}\right) b_{\rho \mu}-\frac{1}{2} b_{\lambda \sigma} \delta a^{\sigma \gamma}\left(\partial_{\gamma} a^{\rho \alpha}\right) b_{\rho \mu}+\frac{1}{2} b_{\lambda \sigma} a^{\alpha \gamma}\left(\partial_{\gamma} \delta a^{\rho \sigma}\right) b_{\rho \mu}+ \\
& -\frac{1}{2} b_{\lambda \sigma} a^{\rho \gamma}\left(\partial_{\gamma} \delta a^{\sigma \alpha}\right) b_{\rho \mu}-\frac{1}{2} b_{\lambda \sigma} a^{\sigma \gamma}\left(\partial_{\gamma} \delta a^{\rho \alpha}\right) b_{\rho \mu}
\end{aligned}
$$

Once we have the explicit expression for the variation of the connection coefficients, we can evaluate the last two terms that appear in the variation of the action (3.36). We start with the first of the two

$$
\begin{aligned}
& -\frac{1}{2} \int_{M} d x \operatorname{Tr}_{V}\left(C^{\mu \nu}{ }_{\alpha} \delta \mathscr{A}^{\alpha}{ }_{\mu \nu}\right)= \\
& =\frac{1}{2} \int_{M} d x \operatorname{Tr}_{V}\left[\mathscr{A}^{\alpha}{ }_{\beta \nu} C^{\mu \nu}{ }_{\alpha} b_{\mu \lambda} \delta a^{\lambda \beta}+b_{\beta \nu} C^{\mu \nu}{ }_{\alpha} \mathscr{A}^{\alpha}{ }_{\mu \lambda} \delta a^{\lambda \beta}+\right. \\
& -\frac{1}{2}\left(\partial_{\gamma} a^{\rho \sigma}\right) b_{\rho \nu} C^{\mu \nu}{ }_{\alpha} b_{\mu \sigma} \delta a^{\alpha \gamma}+\frac{1}{2}\left(\partial_{\gamma} a^{\alpha \sigma}\right) b_{\rho \nu} C^{\mu \nu}{ }_{\alpha} b_{\mu \sigma} \delta a^{\rho \gamma}+ \\
& +\frac{1}{2}\left(\partial_{\gamma} a^{\rho \alpha}\right) b_{\rho \nu} C^{\mu \nu}{ }_{\alpha} b_{\mu \sigma} \delta a^{\sigma \gamma}-\frac{1}{2} b_{\rho \nu} C^{\mu \nu}{ }_{\alpha} b_{\mu \sigma} a^{\alpha \gamma}\left(\partial_{\gamma} \delta a^{\rho \sigma}\right)+ \\
& \left.+\frac{1}{2} b_{\rho \nu} C^{\mu \nu}{ }_{\alpha} b_{\mu \sigma} a^{\rho \gamma}\left(\partial_{\gamma} \delta a^{\alpha \sigma}\right)+\frac{1}{2} b_{\rho \nu} C^{\mu \nu}{ }_{\alpha} b_{\mu \sigma} a^{\sigma \gamma}\left(\partial_{\gamma} \delta a^{\rho \alpha}\right)\right],
\end{aligned}
$$

where in this last expression we used the cyclic property of the trace.

We introduce the following definition, which will be useful in order to simplify the notation,

$$
F_{\beta \alpha \rho}=b_{\beta \nu} C_{\alpha \nu}^{\mu \nu} b_{\mu \rho} .
$$

By using the above definition, the expression in (3.40) can be rewritten as follows

$$
\begin{aligned}
& -\frac{1}{2} \int_{M} d x \operatorname{Tr}_{V}\left(C^{\mu \nu}{ }_{\alpha} \delta \mathscr{A}^{\alpha}{ }_{\mu \nu}\right)= \\
& =\frac{1}{2} \int_{M} d x \operatorname{Tr}_{V}\left[\mathscr{A}^{\alpha}{ }_{\beta \gamma} a^{\gamma \rho} F_{\rho \alpha \lambda} \delta a^{\lambda \beta}+F_{\beta \alpha \rho} a^{\rho \gamma} \mathscr{A}^{\alpha}{ }_{\gamma \lambda} \delta a^{\lambda \beta}+\right. \\
& -\frac{1}{2}\left(\partial_{\gamma} a^{\sigma \rho}\right) F_{\rho \alpha \sigma} \delta a^{\alpha \gamma}+\frac{1}{2}\left(\partial_{\gamma} a^{\alpha \sigma}\right) F_{\rho \alpha \sigma} \delta a^{\rho \gamma}+\frac{1}{2}\left(\partial_{\gamma} a^{\alpha \rho}\right) F_{\rho \alpha \sigma} \delta a^{\sigma \gamma}+ \\
& \left.-\frac{1}{2} F_{\rho \alpha \sigma} a^{\alpha \gamma}\left(\partial_{\gamma} \delta a^{\rho \sigma}\right)+\frac{1}{2} F_{\rho \alpha \sigma} a^{\rho \gamma}\left(\partial_{\gamma} \delta a^{\alpha \sigma}\right)+\frac{1}{2} F_{\rho \alpha \sigma} a^{\sigma \gamma}\left(\partial_{\gamma} \delta a^{\rho \alpha}\right)\right]
\end{aligned}
$$


where the first two terms in the last expression has been derived by using the relation

$$
\mathscr{A}_{\beta \nu}^{\alpha} C^{\mu \nu}{ }_{\alpha} b_{\mu \lambda} \delta a^{\lambda \beta}=\mathscr{A}_{\beta \gamma}^{\alpha} a^{\gamma \rho} b_{\rho \nu} C^{\mu \nu}{ }_{\alpha} b_{\mu \lambda} \delta a^{\lambda \beta}=\mathscr{A}_{\beta \gamma}^{\alpha} a^{\gamma \rho} F_{\rho \alpha \lambda} \delta a^{\lambda \beta} .
$$

By integrating by parts and by relabelling dummy indices we find the final expression for (3.42), namely

$$
\begin{aligned}
-\frac{1}{2} \int_{M} d x \operatorname{Tr}_{V}\left(C^{\mu \nu}{ }_{\alpha} \delta \mathscr{A}^{\alpha}{ }_{\mu \nu}\right) & =\frac{1}{2} \int_{M} d x \operatorname{Tr}_{V}\left[\mathscr{A}_{\beta \gamma}^{\alpha} a^{\gamma \rho} F_{\rho \alpha \gamma}+F_{\beta \alpha \rho} a^{\rho \gamma} \mathscr{A}_{\gamma \lambda}^{\alpha}+\right. \\
& \left.-\frac{1}{2}\left(\partial_{\beta} a^{\rho \sigma}\right)\left(F_{\rho \lambda \sigma}-F_{\lambda \sigma \rho}-F_{\sigma \rho \lambda}\right)\right] \delta a^{\lambda \beta}+ \\
& +\frac{1}{4}\left\{\partial_{\gamma}\left[\left(F_{\rho \lambda \sigma}-F_{\lambda \sigma \rho}-F_{\sigma \rho \lambda}\right) a^{\lambda \gamma}\right]\right\} \delta a^{\rho \sigma}
\end{aligned}
$$

For the last term in the variation of the action (3.36), we use similar arguments which lead us to the expression (3.44). In this case we introduce the following definition:

$$
G_{\beta \alpha \rho}=b_{\beta \alpha} D^{\mu} b_{\mu \rho} .
$$

By using the definition above and the cyclic property of the trace we obtain

$$
\begin{aligned}
& \frac{1}{2} \int_{M} d x \operatorname{Tr}_{V}\left(D^{\mu} \delta \mathscr{A}^{\alpha}{ }_{\mu \alpha}\right)= \\
- & \frac{1}{2} \int_{M} d x \operatorname{Tr}_{V}\left[\mathscr{A}^{\alpha}{ }_{\beta \rho} a^{\rho \gamma} G_{\gamma \alpha \lambda} \delta a^{\lambda \beta}+G_{\beta \alpha \gamma} a^{\gamma \rho} \mathscr{A}^{\alpha}{ }_{\rho \lambda} \delta a^{\lambda \beta}+\right. \\
- & \frac{1}{2}\left(\partial_{\gamma} a^{\rho \sigma}\right) G_{\rho \alpha \sigma} \delta a^{\alpha \gamma}+\frac{1}{2}\left(\partial_{\gamma} a^{\alpha \sigma}\right) G_{\rho \alpha \sigma} \delta a^{\rho \gamma}+\frac{1}{2}\left(\partial_{\gamma} a^{\rho \alpha}\right) G_{\rho \alpha \sigma} \delta a^{\sigma \gamma}+ \\
- & \left.\frac{1}{2} G_{\rho \alpha \sigma} a^{\alpha \gamma}\left(\partial_{\gamma} \delta a^{\rho \sigma}\right)+\frac{1}{2} G_{\rho \alpha \sigma} a^{\rho \gamma}\left(\partial_{\gamma} \delta a^{\alpha \sigma}\right)+\frac{1}{2} G_{\rho \alpha \sigma} a^{\sigma \gamma}\left(\partial_{\gamma} \delta a^{\rho \alpha}\right)\right] .
\end{aligned}
$$

By integrating by parts and relabelling dummy indices we get

$$
\begin{aligned}
\frac{1}{2} \int_{M} d x \operatorname{Tr}_{V}\left(D^{\mu} \delta \mathscr{A}^{\alpha}{ }_{\mu \alpha}\right) & =-\frac{1}{2} \int_{M} d x \operatorname{Tr}_{V}\left[\mathscr{A}_{\beta \rho}^{\alpha} a^{\rho \gamma} G_{\gamma \alpha \lambda}+G_{\beta \alpha \gamma} a^{\rho \gamma} \mathscr{A}_{\rho \lambda}^{\alpha}+\right. \\
& \left.-\frac{1}{2}\left(\partial_{\beta} a^{\rho \sigma}\right)\left(G_{\rho \lambda \sigma}-G_{\lambda \sigma \rho}-G_{\sigma \rho \lambda}\right)\right] \delta a^{\lambda \beta}+ \\
& -\frac{1}{4}\left\{\partial_{\gamma}\left[\left(G_{\rho \lambda \sigma}-G_{\lambda \sigma \rho}-G_{\sigma \rho \lambda}\right) a^{\lambda \gamma}\right]\right\} \delta a^{\rho \sigma}
\end{aligned}
$$


It is worth noticing that in the above expressions, (3.44) and (3.47), the tensor densities $F$ and $G$ always appear in the same combination. This observation justifies the following definitions

$$
X_{\rho \lambda \sigma}=F_{\rho \lambda \sigma}-F_{\lambda \sigma \rho}-F_{\sigma \rho \lambda},
$$

and

$$
Y_{\rho \lambda \sigma}=G_{\rho \lambda \sigma}-G_{\lambda \sigma \rho}-G_{\sigma \rho \lambda} .
$$

By using the two definitions above we can rewrite the arguments of the traces in (3.44) and in (3.47) respectively as

$$
\begin{aligned}
-\frac{1}{2} C^{\mu \nu}{ }_{\alpha} \delta \mathscr{A}^{\alpha}{ }_{\mu \nu} & =\frac{1}{2}\left[\mathscr{A}^{\alpha}{ }_{\beta \gamma} a^{\gamma \rho} F_{\rho \alpha \lambda}+F_{\beta \alpha \rho} a^{\rho \gamma} \mathscr{A}^{\alpha}{ }_{\gamma \lambda}-\frac{1}{2}\left(\partial_{\beta} a^{\rho \sigma}\right) X_{\rho \lambda \sigma}+\right. \\
& \left.+\frac{1}{2} \partial_{\gamma}\left(X_{\lambda \rho \beta} a^{\rho \gamma}\right)\right] \delta a^{\lambda \beta},
\end{aligned}
$$

and

$$
\begin{aligned}
\frac{1}{2} D^{\mu} \delta \mathscr{A}^{\alpha}{ }_{\mu \alpha} & =-\frac{1}{2}\left[\mathscr{A}^{\alpha}{ }_{\beta \rho} a^{\rho \gamma} G_{\gamma \alpha \lambda}+G_{\beta \alpha \gamma} a^{\rho \gamma} \mathscr{A}_{\rho \lambda}^{\alpha}-\frac{1}{2}\left(\partial_{\beta} a^{\rho \sigma}\right) Y_{\rho \lambda \sigma}+\right. \\
& \left.+\frac{1}{2} \partial_{\gamma}\left(Y_{\lambda \rho \beta} a^{\rho \gamma}\right)\right] \delta a^{\lambda \beta} .
\end{aligned}
$$

By combining the results (3.50) and (3.51) we obtain the expression for the last two terms in the variation of the action, namely

$$
\begin{aligned}
& -\frac{1}{2} C^{\mu \nu}{ }_{\alpha} \delta \mathscr{A}^{\alpha}{ }_{\mu \nu}+\frac{1}{2} D^{\mu} \delta \mathscr{A}^{\alpha}{ }_{\mu \alpha}=\frac{1}{2}\left\{\mathscr{A}^{\alpha}{ }_{\beta \rho} a^{\rho \gamma}\left(F_{\gamma \alpha \lambda}-G_{\gamma \alpha \lambda}\right)+\right. \\
& +\left(F_{\beta \alpha \gamma}-G_{\beta \alpha \gamma}\right) a^{\gamma \rho} \mathscr{A}_{\rho \gamma}^{\alpha}+\frac{1}{2}\left(\partial_{\beta} a^{\rho \sigma}\right)\left(Y_{\rho \lambda \sigma}-X_{\rho \lambda \sigma}\right)+ \\
& \left.-\frac{1}{2} \partial_{\gamma}\left[\left(Y_{\lambda \rho \beta}-X_{\lambda \rho \beta}\right) a^{\rho \gamma}\right]\right\} \delta a^{\lambda \beta} \text {. }
\end{aligned}
$$

\section{Noncommutative Einstein Equations}

With the expression (3.52) for the last two terms in (3.36), the variation of the action has the form (3.27) which is suitable for the derivation of the dynamical equations of the model. Before writing the complete dynamical equations, we will simplify further the expression (3.52). 
The definition (3.48) gives a linear relation between the matrix-valued tensor density $X$ and a particular combination of matrix-valued tensor density $F$, a similar linear relation between $Y$ and $G$ is given in (3.49). By using simple tensor algebra, it can be easily shown that those relations can be inverted, namely we can write

$$
F_{\rho \lambda \sigma}=-\frac{1}{2}\left(X_{\lambda \sigma \rho}+X_{\sigma \rho \lambda}\right)
$$

and

$$
G_{\rho \lambda \sigma}=-\frac{1}{2}\left(Y_{\lambda \sigma \rho}-Y_{\sigma \rho \lambda}\right) .
$$

By substituting the equations (4.53) and (4.54) in the expression (3.52) we obtain the following

$$
\begin{aligned}
-\frac{1}{2} C_{\alpha}^{\mu \nu} \delta \mathscr{A}^{\alpha}{ }_{\mu \nu} & +\frac{1}{2} D^{\mu} \delta \mathscr{A}^{\alpha}{ }_{\mu \alpha}=\frac{1}{4}\left\{\mathscr{A}_{\beta \rho}^{\alpha} a^{\rho \gamma}\left[\left(Y_{\alpha \lambda \gamma}-X_{\alpha \lambda \gamma}\right)+\left(Y_{\lambda \gamma \alpha}-X_{\lambda \gamma \alpha}\right)\right]+\right. \\
+ & {\left[\left(Y_{\alpha \gamma \lambda}-X_{\alpha \gamma \lambda}\right)+\left(Y_{\gamma \beta \alpha}-X_{\gamma \beta \alpha}\right)\right] a^{\rho \gamma} \mathscr{A}_{\rho \lambda}^{\alpha}+} \\
+ & \left.\left(\partial_{\beta} a^{\rho \sigma}\right)\left(Y_{\rho \lambda \sigma}-X_{\rho \lambda \sigma}\right)-\partial_{\gamma}\left[\left(Y_{\lambda \rho \beta}-X_{\lambda \rho \beta}\right) a^{\rho \gamma}\right]\right\} \delta a^{\lambda \beta}
\end{aligned}
$$

We can see, in the last formula, that the tensor densities $X$ and $Y$ enter always in the same combination. It is useful, therefore, to define the following tensor density

$$
H_{\mu \nu \rho}=Y_{\mu \nu \rho}-X_{\mu \nu \rho} .
$$

With this last definition we can rewrite (4.55) as

$$
\begin{aligned}
& -\frac{1}{2} C_{\alpha}^{\mu \nu} \delta \mathscr{A}^{\alpha}{ }_{\mu \nu}+\frac{1}{2} D^{\mu} \delta \mathscr{A}^{\alpha}{ }_{\mu \alpha}=\frac{1}{4}\left[\mathscr{A}_{\beta \rho}^{\alpha} a^{\rho \gamma} H_{\alpha \lambda \gamma}+\mathscr{A}_{\beta \rho}^{\alpha} a^{\rho \gamma} H_{\lambda \gamma \alpha}+\right. \\
& \left.+H_{\alpha \gamma \beta} a^{\rho \gamma} \mathscr{A}^{\alpha}{ }_{\rho \lambda}+H_{\gamma \beta \alpha} a^{\rho \gamma} \mathscr{A}^{\alpha}{ }_{\rho \lambda}+\left(\partial_{\beta} a^{\rho \sigma}\right) H_{\rho \lambda \sigma}-\partial_{\gamma}\left(H_{\lambda \rho \beta} a^{\rho \gamma}\right)\right] \delta a^{\lambda \beta} .
\end{aligned}
$$

By using the compatibility condition (2.15), we can write that

$$
\partial_{\beta} a^{\rho \sigma}=-\mathscr{A}^{\rho}{ }_{\gamma \beta} a^{\gamma \sigma}-\mathscr{A}^{\sigma}{ }_{\gamma \beta} a^{\rho \gamma},
$$

moreover we obtain that

$$
-\partial_{\gamma}\left(H_{\lambda \rho \beta} a^{\rho \gamma}\right)=-\left(\partial_{\gamma} H_{\lambda \rho \beta}\right) a^{\rho \gamma}+H_{\lambda \rho \beta} \mathscr{A}_{\sigma \gamma}^{\rho} a^{\sigma \gamma}+H_{\lambda \rho \beta} \mathscr{A}_{\sigma \gamma}^{\gamma} a^{\sigma \rho} .
$$

Since $H_{\mu \nu \rho}$ is a tensor density, we can write

$$
\mathcal{D}_{\gamma} H_{\lambda \rho \beta}=\partial_{\gamma} H_{\lambda \rho \beta}-\mathscr{A}^{\alpha}{ }_{\lambda \gamma} H_{\alpha \rho \beta}-\mathscr{A}^{\alpha}{ }_{\rho \gamma} H_{\lambda \alpha \beta}-\mathscr{A}_{\beta \gamma}^{\alpha} H_{\lambda \rho \alpha}-\mathscr{A}^{\alpha}{ }_{\gamma \alpha} H_{\lambda \rho \beta} .
$$


By using the results obtained in (4.58), (4.59) and (4.60) we can express (4.57) as follows

$$
\begin{aligned}
& -\frac{1}{2} C^{\mu \nu}{ }_{\alpha} \delta \mathscr{A}^{\alpha}{ }_{\mu \nu}+\frac{1}{2} D^{\mu} \delta \mathscr{A}^{\alpha}{ }_{\mu \alpha}=\frac{1}{4}\left\{2 \mathscr{A}^{\alpha}{ }_{[\beta \rho]} a^{\rho \gamma} H_{\alpha \lambda \gamma}+2 H_{\alpha \lambda \beta} a^{\rho \gamma} \mathscr{A}^{\alpha}{ }_{[\rho \lambda]}+\right. \\
& -\left(\mathcal{D}_{\gamma} H_{\lambda \rho \beta}\right) a^{\rho \gamma}-\left[\mathscr{A}_{\lambda \rho}^{\alpha}, H_{\alpha \gamma \beta}\right] a^{\rho \gamma}-H_{\alpha \gamma \beta}\left[\mathscr{A}^{\alpha}{ }_{\lambda \rho}, a^{\rho \gamma}\right]-\left[\mathscr{A}^{\alpha}{ }_{\rho \gamma}, H_{\lambda \alpha \beta}\right] a^{\rho \gamma}+ \\
& \left.-\left[\mathscr{A}^{\alpha}{ }_{\gamma \alpha}, H_{\lambda \rho \beta}\right] a^{\rho \gamma}-\mathscr{A}_{\beta \gamma}^{\alpha}\left[H_{\lambda \rho \alpha}, a^{\rho \gamma}\right]\right\} \delta a^{\lambda \beta} \text {. }
\end{aligned}
$$

At this point we introduce the operator $P$ defined as

$$
P_{\gamma} H_{\lambda \rho \beta}=\mathcal{D} H_{\lambda \rho \beta}+\left[\mathscr{A}^{\alpha}{ }_{\lambda \gamma}, H_{\alpha \rho \beta}\right]+\left[\mathscr{A}^{\alpha}{ }_{\rho \gamma}, H_{\lambda \alpha \beta}\right]+\left[\mathscr{A}_{\beta \gamma}^{\alpha}, H_{\lambda \rho \alpha}\right]+\left[\mathscr{A}^{\alpha}{ }_{\gamma \alpha}, H_{\lambda \rho \beta}\right] .
$$

By using the last definition in (4.61) one obtains

$$
\begin{gathered}
-\frac{1}{2} C^{\mu v}{ }_{\alpha} \delta \mathscr{A}^{\alpha}{ }_{\mu \nu}+\frac{1}{2} D^{\mu} \delta \mathscr{A}^{\alpha}{ }_{\mu \alpha}=\frac{1}{4}\left\{2 \mathscr{A}^{\alpha}{ }_{[\beta \rho]} a^{\rho \gamma} H_{\alpha \lambda \gamma}+2 H_{\alpha \lambda \beta} a^{\rho \gamma} \mathscr{A}^{\alpha}{ }_{[\rho \lambda]}+\right. \\
\left.-\left(P_{\gamma} H_{\lambda \rho \beta}\right) a^{\rho \gamma}+\left[\mathscr{A}^{\alpha}{ }_{\beta \gamma}, H_{\lambda \rho \alpha}\right] a^{\rho \gamma}-H_{\alpha \gamma \beta}\left[\mathscr{A}^{\alpha}{ }_{\lambda \rho}, a^{\rho \gamma}\right]-\mathscr{A}_{\beta \gamma}^{\alpha}\left[H_{\lambda \rho \alpha}, a^{\rho \gamma}\right]\right\} \delta a^{\lambda \beta} .
\end{gathered}
$$

We finally have all the ingredients that we need in order to write the dynamical equations of the theory. Now we only have to find an expression for the variation $\delta \rho$. The definition of $\rho$ is given in (2.21), and its variation can be straightforwardly evaluated as follows

$$
\delta \rho=-\int_{\mathbb{R}^{n}} \frac{d \xi}{\pi^{\frac{n}{2}}} \int_{0}^{1} d s e^{-(1-s) A(\xi)} \delta a^{\mu v} \xi_{\mu} \xi_{\nu} e^{-s A(\xi)},
$$

where

$$
A(\xi)=a^{\mu v} \xi_{\mu} \xi_{v} .
$$

Once we have the expression (4.64) for the variation, we can use the cyclic property of the trace to write that

$$
\operatorname{Tr}_{V}(\delta \rho \mathcal{R})=\operatorname{Tr}_{V}\left[-\int_{\mathbb{R}^{n}} \frac{d \xi}{\pi^{\frac{n}{2}}} \int_{0}^{1} d s e^{-s A(\xi)} \mathcal{R} e^{-(1-s) A(\xi)} \xi_{\mu} \xi_{v}\right] \delta a^{\mu \nu} .
$$

By combining (4.66), (4.63) and (3.36) we obtain the noncommutative Einstein equations in absence of matter, namely

$$
\mathcal{G}_{\mu \nu}=0,
$$


where

$$
\begin{aligned}
\mathcal{G}_{\mu \nu} & =\frac{1}{2}\left\{\rho, \mathcal{R}_{\mu \nu}\right\}+\mathcal{F}_{\mu \nu}+\frac{1}{2} \mathscr{A}^{\alpha}{ }_{[\mu \rho]} a^{\rho \gamma} H_{\alpha \nu \gamma}+\frac{1}{2} H_{\alpha \lambda \nu} a^{\rho \gamma} \mathscr{A}^{\alpha}{ }_{[\rho \mu]}-\frac{1}{4}\left(P_{\gamma} H_{\mu \rho v}\right) a^{\rho \gamma}+ \\
& +\frac{1}{4}\left[\mathscr{A}^{\alpha}{ }_{\nu \gamma}, H_{\mu \rho \alpha}\right] a^{\rho \gamma}-\frac{1}{4} H_{\alpha \gamma \nu}\left[\mathscr{A}^{\alpha}{ }_{\mu \rho}, a^{\rho \gamma}\right]-\frac{1}{4} \mathscr{A}^{\alpha}{ }_{\nu \gamma}\left[H_{\mu \rho \alpha}, a^{\rho \gamma}\right]
\end{aligned}
$$

is the noncommutative Einstein tensor, $\mathcal{F}_{\mu \nu}$ is defined by

$$
\mathcal{F}_{\mu \nu}=-\int_{\mathbb{R}^{n}} \frac{d \xi}{\pi^{\frac{n}{2}}} \int_{0}^{1} d s e^{-s A(\xi)} \mathcal{R} e^{-(1-s) A(\xi)} \xi_{\mu} \xi_{\nu},
$$

and the tensor density $H$ has the explicit form

$$
H_{\alpha \lambda \gamma}=b_{\alpha v}\left(\delta_{\lambda}^{v} D^{\mu}-C_{\lambda}^{\mu \nu}\right) b_{\mu \gamma}-b_{\lambda v}\left(\delta_{\gamma}^{v} D^{\mu}-C_{\gamma}^{\mu v}\right) b_{\mu \alpha}-b_{\gamma \nu}\left(\delta_{\alpha}^{v} D^{\mu}-C_{\alpha}^{\mu v}\right) b_{\mu \lambda} .
$$

These equations are the main result of the present paper. One can show that the first two terms in the equations (4.68) represent a straightforward generalization of Einstein's equation to endomorphism-valued objects and the rest of the terms can be considered as a genuine noncommutative part which is not present in Einstein's equation. It is interesting to note that the pure noncommutative part is completely described by the tensor density $H_{\mu v \rho}$ defined in (4.70).

Moreover the equation (4.67) satisfies the requirement (3.29), which, in words, expresses the necessity that our model reduces, in the commutative limit, to the standard theory of general relativity. In fact, the trace of the pure noncommutative terms vanishes, because of the presence of the commutators, and the first two terms just give

$$
\frac{1}{N} \operatorname{Tr}_{V}\left(\frac{1}{2}\left\{\rho, \mathcal{R}_{\mu \nu}\right\}+\mathcal{F}_{\mu \nu}\right)=\sqrt{g}\left(R_{\mu \nu}-\frac{1}{2} g_{\mu \nu} R\right) .
$$

For an arbitrary matrix algebra the equation (4.67) becomes more complicated than the ordinary Einstein's equation due to presence of the new tensor density $H_{\mu \nu \rho}$. We mention, now, a particular case in which (4.67) simplifies. The formalism used so far deals with geometric quantities which are endomorphism-valued, namely they take values in $\operatorname{End}(V)$. By choosing a basis in the vector space $V$ we can represent $\operatorname{End}(V)$ by means of matrices. Let us suppose that the algebra under consideration is Abelian, in this case all the elements commute with each other and the tensor density $H_{\mu \nu \rho}$ vanishes identically and the equation (4.67) becomes

$$
\mathcal{R}_{\mu \nu}-\frac{1}{2} b_{\mu \nu} \mathcal{R}=0
$$


Therefore, in case of a commutative matrix algebra, the equation of motion of our model have the same form as Einstein's equation, with the only difference that (4.72) is matrix-valued.

\section{The Action for the Matter Field}

In order to have a complete theory for the gravitational field we need to describe the dynamics of the matter field in the framework of matrix general relativity. The main idea is to extend the general results of classical field theory. We will consider, in the following, the dynamics of a multiplet of free scalar fields propagating on a manifold $M$. We can construct an invariant action by using the matrix valued metric $a^{\mu v}$ and the measure $\rho$. A typical action is

$$
S_{\text {matter }}(a, \varphi)=\frac{1}{4} \int_{M} d x\left\{-\left\langle\partial_{\mu} \varphi,\left\{\rho, a^{\mu \nu}\right\} \partial_{\nu} \varphi\right\rangle-\langle\varphi,\{\rho, Q\} \varphi\rangle\right\},
$$

where $\langle$,$\rangle denotes the fiber inner product on the vector bundle V$, and $Q$ is a constant mass matrix determining the masses of the scalar fields. The equations of motion of the scalar fields are then obviously

$$
\left[-\partial_{\mu}\left\{\rho, a^{\mu v}\right\} \partial_{v}+\{\rho, Q\}\right] \varphi=0
$$

The complete action of the gravity and matter is described then by

$$
S(a, \varphi)=S_{\mathrm{MGR}}(a)+S_{\text {matter }}(a, \varphi) .
$$

By varying the above action with respect to $a^{\mu \nu}$ one obtains the noncommutative Einstein equation in presence of matter

$$
\mathcal{G}_{\mu \nu}=8 \pi G N \mathcal{T}_{\mu \nu}
$$

where $\mathcal{T}_{\mu \nu}$ is the matrix energy-momentum tensor defined by

$$
\mathcal{T}_{\mu \nu}=-\frac{1}{2} \frac{\delta S_{\text {matter }}}{\delta a^{\mu \nu}}
$$

By using the explicit lagrangian (5.73) for the matter field, we obtain the expression for the energy-momentum tensor

$$
\mathcal{T}_{\mu \nu}=\frac{1}{8}\left[\left\{\rho, \partial_{\mu} \varphi \otimes \partial_{\nu} \varphi\right\}+\mathcal{M}_{\mu \nu}+\mathcal{N}_{\mu \nu}\right]+(\mu \leftrightarrow v),
$$


where the explicit form of $\mathcal{M}_{\mu \nu}$ and $\mathcal{N}_{\mu \nu}$ is obtained by using the variation of the scalar density $\rho$ in (4.64), namely

$$
\mathcal{M}_{\mu \nu}=-\int_{\mathbb{R}^{n}} \frac{d \xi}{\pi^{\frac{n}{2}}} \int_{0}^{1} d s e^{-s A(\xi)}\left\{a^{\alpha \beta}, \partial_{\alpha} \varphi \otimes \partial_{\beta} \varphi\right\} e^{-(1-s) A(\xi)} \xi_{\mu} \xi_{v}
$$

and

$$
\mathcal{N}_{\mu \nu}=-\int_{\mathbb{R}^{n}} \frac{d \xi}{\pi^{\frac{n}{2}}} \int_{0}^{1} d s e^{-s A(\xi)}\{Q, \varphi \otimes \varphi\} e^{-(1-s) A(\xi)} \xi_{\mu} \xi_{\nu} .
$$

It is worth remarking, here, that the above formula (5.78) for the energymomentum tensor $\mathcal{T}_{\mu \nu}$ reduces, in the commutative limit, to the standard result, e.g. [5].

\section{Conclusions}

The main idea of this new model is to describe the gravitational field by a multiplet of gauge fields with some internal structure. For this purpose the metric field $g^{\mu \nu}$, which describes gravity in general relativity, is replaced by a matrix-valued 2-tensor field $a^{\mu \nu}$. This allows the model to have a much richer content in describing gravitational phenomena. A more general geometric picture is developed by allowing the metric to be matrix-valued. Most of the geometric quantities, used in describing gravity, can be generalized to be endomorphism-valued. In this framework it is possible to introduce an action for the gravitational field which is diffeomorphisms and gauge invariant, that leads, after performing the variation with respect to $a^{\mu \nu}$, to the modified (noncommutative) Einstein equation. It is interesting that the noncommutative part of the modified equations only depends on a specific tensor density $H_{\mu \nu \rho}$ and on a linear combination of its commutators.

With an explicit expression for the noncommutative Einstein equation, it will be possible to study some particular simple solutions of (4.67); for example a static and spherically symmetric solution, which would describe the gravitational field outside a spherically symmetric massive body, or a spherically symmetric homogeneous solution, that would describe noncommutative cosmological models. These simple examples are very interesting and we plan to study them systematically elsewhere.

We would like to make a final remark. In our model all the geometric quantities that we need to develop the formalism are endomorphism-valued. Once a 
basis for the vector bundle $V$ has been fixed, we can represent elements of $\operatorname{End}(V)$ by matrices. Of course the description of physical phenomena has to be independent from the particular realization of the representation. This is, ultimately, related to the gauge invariance of the theory. We believe that by an opportune choice of gauge, namely an opportune representation of End $(V)$ by matrices, the dynamical equation (4.67) could be simplified further. The search for such particular gauge, if it exists, requires further studies in matrix differential geometry and matrix general relativity.

\section{References}

[1] Avramidi I G 2004 Matrix General Relativity: a new look at old problems Class. and Quantum Grav. 21 103-120

[2] Avramidi I G 2004 Gauged Gravity via Spectral Asymptotics of non-Laplace type Operators Journal of High Energy Physics 07030

[3] Mann R B 1984 Five theories of Gravity Class. and Quantum Grav. 1 561572

[4] Wald R M 1987 A new type of Gauge invariance for a collection of massless spin-2 fields: II. Geometrical interpretation Class. and Quantum Grav. 4 $1279-1316$

[5] Fulling S A 1989 Aspects of Quantum Field Theory in Curved Space-Time (Cambridge: Cambridge University Press)

[6] Konechny A and Schwarz A 2002 Introduction to M(atrix) theory and noncommutative geometry, Phys. Rep. $360353-465$

[7] Madore J and Mourad J 1994 A noncommutative extension of gravity Int. J. Mod. Phys. D 3 221-224

[8] Madore J 1999 An Introduction to Noncommutative Differential Geometry and its Physicsl Applications (Cambridge: Cambridge University Press)

[9] Harikumar E and Rivelles V O 2006 Noncommutative Gravity, Class.Quant.Grav. 23 7551-7560 